\title{
Numerical Analysis of the EM Field from a Moving Source and the Application for a Moving Vehicle
}

\author{
Tatsuya Akata \\ Graduate School of Bionics, \\ Computer and Media Sciences \\ Tokyo University of Technology \\ Tokyo, Japan \\ g211200104@edu.teu.ac.jp
}

\author{
Shafrida Sahrani \\ Faculty of Engineering \\ Universiti Malaysia Sarawak \\ Kota Samarahan, Malaysia \\ sshafrida@feng.unimas.my
}

\author{
Michiko Kuroda \\ School of Computer Science \\ Tokyo University of Technology \\ Tokyo, Japan \\ kuroda@stf.teu.ac.jp
}

\begin{abstract}
Many practical engineering applications require the numerical solution for the analysis of the EM field by a moving source and/or a moving body. We have previously proposed the Overset Grid Generation method coupled with FDTD method for the analysis of the EM field with moving boundaries considering Doppler Effect. By overlapping one moving sub-mesh on a static main mesh, each mesh is calculated alternately by using interpolation technique. For higher velocity value, Lorentz transformation is applied to the FDTD method. In this paper, after verifying the accuracy of this technique, it is applied for the EM field at an intersection when the input source is moving towards the moving vehicle.
\end{abstract}

Keywords-FDTD method; Lorentz transformation; moving boundary problems; moving source; Inter-vehicle communication; Overset Grid Generation method.

\section{INTRODUCTION}

In recent years, the analysis of the electromagnetic (EM) field around a moving body is of importance to solve various problems arising in the engineering applications [1]-[3]. More recently, the solution of EM wave scattering problem remains a crucial element for the realization of next generation nano electronic devices which involve complicated geometries with complex and dynamic moving boundary [4],[5]. Hence, current and the future of some technologies crucially depend on advanced algorithm in the numerical modeling technique with powerful computational capabilities for analyzing the EM field with a complex moving body particularly at high velocities. The Overset Grid Generation method combined with Finite-Difference Time-Domain (FDTD) method and Lorentz transformation has been previously proposed to solve the EM field from a moving body for higher velocity cases $[6],[7]$.

The Overset Grid Generation method has been used mainly in the computational fluid dynamics (CFD) [8]. This method consists of a static main mesh and a moving sub-mesh that overlapped each another. It can reduce the geometrically complex problem to a simple set of grids. The proposed numerical technique aims to find a solution to keep the submesh moving with high velocity value. According to the special relativity, Lorentz transformation requires at least two frames of reference that move with relative velocity [9].
The FDTD method is widely used to solve the EM field problems due to its low computational complexity [10]. Moreover, this method is very easy to implement in any computing environment including on the structured grids. The time components for the FDTD method are obtained at alternate half-time steps. However, the time component in Lorentz transformation changes at each grid point. Here, the time components that were changed in Lorentz transformation are fixed by using linear interpolation scheme in the Overset Grid Generation method. This allows a coherent point in time component with the FDTD method, which is an important element of this proposed numerical technique.

In this paper, the proposed Overset Grid Generation method combined with the FDTD method and Lorentz transformation is applied to the analysis of the EM field when the input source is moving towards the moving body. The accuracy of the proposed method is verified. Then, the characteristics of the EM field at the intersection when the source and vehicle is moving are observed. The numerical results of the moving case are compared with the stationary case.

\section{LORENTZ TRANSFORMATION FOR FDTD METHOD}

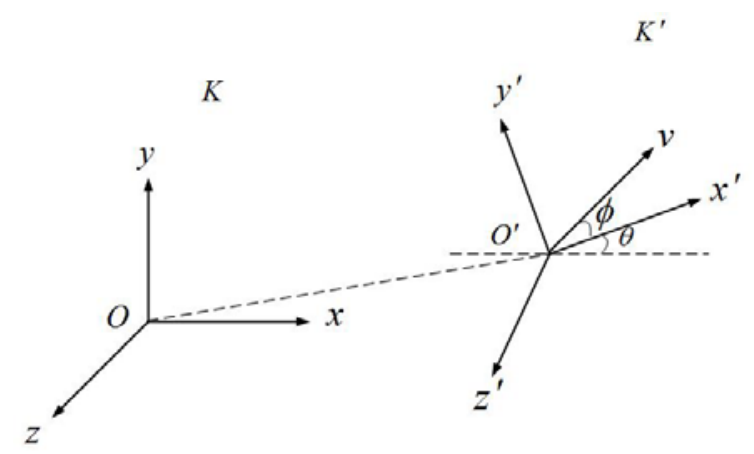

Fig. 1. Two inertial frames in relative motion.

Fig. 1 shows the basic Lorentz transformation in two inertial frames and move relative to one another [9]. Consider two observers, $O$ and $O^{\prime}$ in frame $K$ and $K^{\prime}$ starts at the initial time $t=t^{\prime}=0$, respectively. The observer $O^{\prime}$ in $\left(x^{\prime}, y^{\prime}, z^{\prime}, t^{\prime}\right)$ 